\title{
Traumatic rupture of the thoracic aorta: Ten years of delayed management
}

\author{
Davide Pacini, $M D^{\mathrm{a}}$ \\ Emanuela Angeli, MDa \\ Rossella Fattori, $M D^{b}$ \\ Luigi Lovato, $\mathrm{MD}^{\mathrm{b}}$ \\ Guido Rocchi, MD ${ }^{\mathrm{C}}$ \\ Luca Di Marco, MD \\ Marcello Bergonzini, MD \\ Giovanni Grillone, $\mathrm{MD}^{\mathrm{d}}$ \\ Roberto Di Bartolomeo, MD ${ }^{\mathrm{a}}$
}

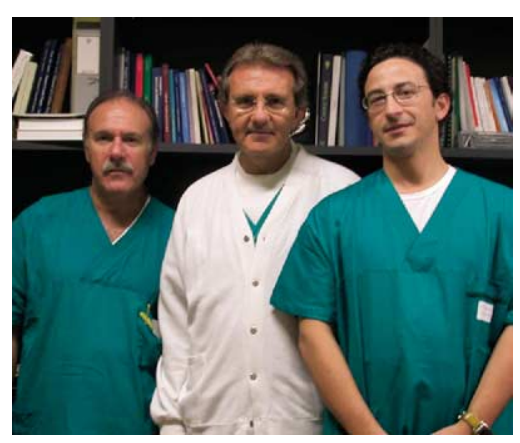

Grillone, Di Bartolomeo, Pacini (left to right)
Objective: Traumatic rupture of the thoracic aorta is a highly fatal condition in which patient outcome is strongly conditioned by other associated injuries. Delayed aortic treatment has been proposed to improve results.

Methods: The charts of 69 patients with traumatic rupture of the thoracic aorta observed between 1980 and 2003 were reviewed. Patients were grouped according the timing of repair: group I, immediate repair (21 patients); and group II, delayed repair (48 patients). In group II, 45 patients were treated surgically or by endovascular procedure.

Results: In-hospital mortalities were 4 of 21 patients (19\%) in group I and 2 of 48 patients (4.2\%) in group II. There were 3 cases of paraplegia in group I and none in group II.

Conclusion: Improvement of patient outcome with traumatic rupture of the thoracic aorta can be achieved by delaying surgical repair until after management of major associated injuries if there are no signs of impending rupture. Endovascular treatment is feasible and safe and may represent a valid alternative to open surgery in selected cases.

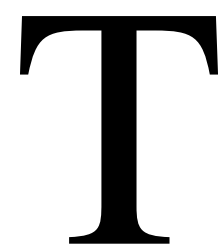

raumatic rupture of the thoracic aorta (TRTA) is a life-threatening lesion. It occurs in $10 \%$ to $30 \%$ of fatalities from blunt thoracic trauma and is the second most common cause of death after head injury. Generally, about $80 \%$ of affected patients do not survive to reach the hospital. Despite rapid transportation to trauma centers, aggressive resuscitation, and emergency surgical repair, in-hospital mortality remains high, ranging from $15 \%$ to $28 \% .^{1-4}$ This high mortality is linked, for the most part, to the associated lesions. New strategies in the surgical timing of TRTA should be considered in an attempt to modify its negative prognosis. The aim of this retrospective study was to examine the evolution of the management of TRTA in our department.

\section{Methods}

We reviewed the medical records of 69 patients with acute TRTA who were admitted to S. Orsola-Malpighi Hospital between 1980 and 2003. Twenty seven patients (39.1\%) were admitted to our hospital directly from the scene of the accident, and $42(60.9 \%)$ were transferred from regional trauma centers. 
TABLE 1. Associated injuries

\begin{tabular}{lcc}
\hline Associated injuries & No. & $\%$ \\
\hline Thoracic lesions & $46 / 69$ & $66.7 \%$ \\
Rib fracture & 35 & \\
Sternal fracture & 2 & \\
Pulmonary contusion & 39 & \\
Hemopericardium & 1 & $23.2 \%$ \\
Head injuries & $16 / 69$ & $78.3 \%$ \\
Orthopedic injuries & $54 / 69$ & \\
Lower limb fracture & 25 & \\
Upper limb fracture & 14 & \\
Pelvic fracture & 17 & \\
Rachis fracture & 2 & \\
Maxillofacial fracture & 7 & \\
Abdominal lesions & $19 / 69$ & \\
Liver laceration or contusion & 8 & \\
Kidney contusion & 5 & \\
Spleen rupture & 9 & \\
Diaphragmatic rupture & 1 & \\
Bladder rupture & 2 & \\
Intestinal rupture & 1 & \\
\hline
\end{tabular}

The patients were divided into two groups according to time period and type of management: group I (21 patients) consisted of those from 1980 to 1992 treated on an emergency basis, and group II (48 patients) consisted of those from 1993 to 2003 managed with delayed aortic repair. Group I, which was previously reported on in detail, ${ }^{5}$ comprised 21 patients with a mean age of 31.2 years (range 6-66 years). Group II comprised 48 patients ranging in age from 15 to 66 years (mean 38.3 years). All patients had been in violent accidents involving a mechanism of sudden deceleration: car crashes in 67 cases and work-related accidents involving falls in 2 cases.

The diagnosis of aortic injury was suggested by chest radiography and confirmed in group I by aortography and in group II by computed tomographic (CT) scan in 48 cases, magnetic resonance imaging in 28 cases, and transesophageal echocardiography in 5 cases. At present, spiral CT scan represents the only imaging modality used for the diagnosis of TRTA.

The site of the TRTA was the isthmus in 64 patients, the descending thoracic aorta distal to the isthmus in 3 patients, and the posterior part of the aortic arch in 2 patients. The aortic lesion was isolated in only 5 patients $(7.2 \%)$. All other patients sustained major associated injuries as summarized in Table 1.

\section{Patient Management}

In group I, surgical repair was performed immediately after the diagnosis of TRTA. In group II, the treatment of the TRTA was delayed until after intensive medical therapy and treatment of the associated lesions.

The intensive medical treatment, as previously reported, ${ }^{5}$ can be summarized as follows. The patients were admitted to the intensive care unit and immediately underwent intensive resuscitation with continuous monitoring of electrocardiogram, arterial blood pressure (radial or femoral arteries), central venous pressure, and vital signs. Conservative treatment consisted primarily of the administration of
TABLE 2. Emergency treatment of associated injuries in group II

\begin{tabular}{lrr}
\hline Treatment & No. & $\%$ \\
\hline Bone fracture stabilization & $39 / 48$ & $81.3 \%$ \\
Splenectomy & $9 / 48$ & $18.8 \%$ \\
Liver trauma repair & $4 / 48$ & $8.4 \%$ \\
Bladder repair & $2 / 48$ & $4.2 \%$ \\
Nephrectomy & $2 / 48$ & $4.2 \%$ \\
Cholecystectomy & $1 / 48$ & $2.1 \%$ \\
Intestinal resection & $1 / 48$ & $2.1 \%$ \\
Diaphragm repair & $1 / 48$ & $2.1 \%$ \\
Pericardiocentesis & $1 / 48$ & $2.1 \%$ \\
Posterior vertebral laminectomy & $1 / 48$ & $2.1 \%$ \\
\hline
\end{tabular}

TABLE 3. Techniques of surgical management

\begin{tabular}{lcc}
\hline Technique & Group I & Group II \\
\hline Clamp and sew & $10 / 21(47.6 \%)$ & - \\
Passive shunt & $1 / 21(4.8) \%$ & - \\
Total CPB & $3 / 21(14.3 \%)$ & $2 / 30(6.7 \%)$ \\
Left heart bypass & $7 / 21(33.3 \%)$ & $28 / 30(93.3 \%)$ \\
\hline
\end{tabular}

$\beta$-blockers (metoprolol) and vasodilators (sodium nitroprusside, calcium-channel blockers, and nitrates), often in combination, to maintain a systolic blood pressure of about $100 \mathrm{~mm} \mathrm{Hg}$. Thirtyeight patients $(79.2 \%)$ required endotracheal intubation and mechanical ventilation, and in 21 patients a chest tube was inserted to drain a hemothorax or pleural effusion. Drainage of more than $1500 \mathrm{~mL}$ was obtained from only 1 patient. Twenty eight patients $(58.3 \%)$ needed blood transfusion. Once the patient was hemodynamically stable (systolic blood pressure $80-100 \mathrm{~mm} \mathrm{Hg}$, adequate urinary output, and heart rate $50-80$ beats/min), antihypertensive therapy was given orally and continued for the whole period before aortic repair.

Magnetic resonance imaging or, more recently, multidetector CT scan was performed at 7,15 , and 30 days to monitor the evolution of posttraumatic aneurysm, periaortic hematoma, mediastinal hematoma, and thoracic associated lesions. All major associated injuries were treated surgically before repair of the aorta when the aortic lesion was stable (Table 2). The TRTA was considered stable in the absence of such signs of an impending rupture as uncontrolled blood pressure, repeated hemothorax $(>1000 \mathrm{~mL})$, circumferential lesion, contrast medium extravasation on CT images, and pseudocoarctation syndrome. Aortic repair was thus planned after the resolution of all other significant associated injuries.

In group II, 5 patients required urgent aortic repair: 3 underwent surgery and 2 underwent endovascular procedures. In 3 cases, the aortic repair was performed because of a massive hemothorax with contrast media extravasation on CT scan (8 to 36 hours from the trauma), in 1 because of a rapid enlargement of the pseudoaneurysm (12 $\mathrm{mm}$ at 1 month after the trauma), and in 1 for pseudocoarctation syndrome ( 28 hours after the trauma). 


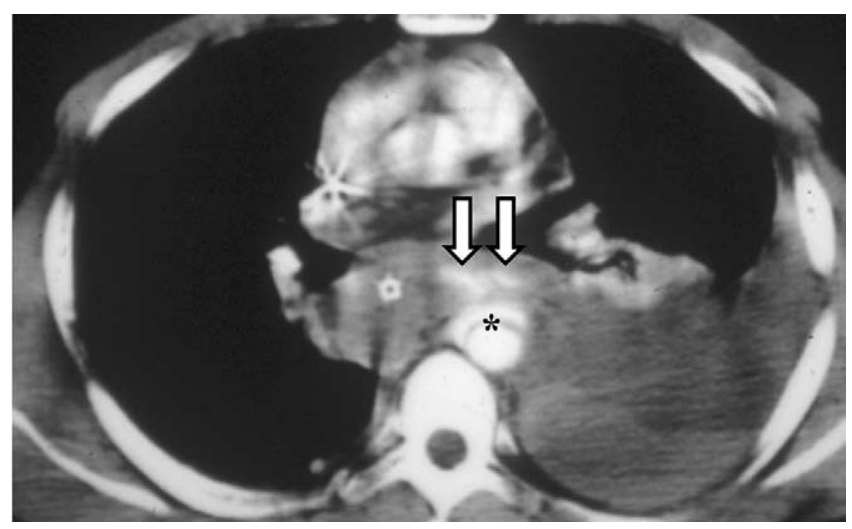

Figure 1. Chest CT scan shows isthmic aortic rupture (asterisk) with massive left hemothorax and contrast media extravasation (arrows).

\section{TABLE 4. Postoperative complications}

\begin{tabular}{lcc}
\hline Complication & Group I & Group II \\
\hline Paraplegia & $3 / 21(14.3 \%)$ & - \\
Paraparesis & $1 / 21(4.8 \%)$ & - \\
Acute renal failure & $1 / 21(4.8 \%)$ & - \\
Coma & $1 / 21(4.8 \%)$ & - \\
Bleeding & $1 / 21(4.8) \%$ & - \\
Chylothorax & - & $1 / 30(3.3 \%)$ \\
Left recurrent nerve injury & $3 / 21(14.3 \%)$ & $4 / 30(13.4 \%)$ \\
Pericarditis & - & $2 / 30(6.7 \%)$ \\
\hline
\end{tabular}

All complications occurred in surgically treated patients.

The surgical techniques consisted of graft replacement of the ruptured segment of the aorta in 49 cases and direct suturing of the aortic lesion in 2 cases. The techniques which used as an adjunct to surgery were clamp and sew, shunts, total cardiopulmonary bypass (CPB), and left heart bypass, as summarized in Table 3. Because a centrifugal pump was available, spinal cord protection and distal organs perfusion were achieved by the use of left heart by pass whenever possible ( 35 patients, $50.7 \%$ ). In 5 cases, the aortic repair had to be carried out during circulatory arrest with deep hypothermia because of the proximal extension of the aortic tear into the transverse arch.

\section{Endovascular Repair}

Aortic injury was managed with an endoluminal stent graft in 15 patients: 2 in emergency and 13 delayed. Adequate anatomic criteria for stent-graft placement included a rupture located distal to the left subclavian artery with the proximal neck of the healthy aorta $5 \mathrm{~mm}$ or more in length, absence of thrombus in the fixation regions, and proper peripheral vascular access. Three patients were considered ineligible for stent grafting and were surgically treated: $2 \mathrm{had}$ an inadequate proximal neck, and 1 had pseudocoarctation syndrome. All endovascular procedures were performed in the operating theater and monitored by a portable radiographic C-arm system with digital subtraction angiography and by echocardiography with a multiplanar transesophageal probe. At the beginning of the procedures, heparin (5000 units) was used only in the delayed cases, whereas no systemic heparin was administered in the 2 acute cases.

\section{Results}

In group I, the 21 patients underwent aortic repair with a mean interval from accident to operation of 8 hours. Four (19\%) died: 3 during the operation and 1 after 30 days. The causes of death were massive bleeding in 2 patients, cardiac arrest in 1, and septic shock in 1 .

In group II, the mean intensive care unit stay after the trauma was 12 days (range 1-60 days). The average time from injury to the aortic repair was $3.4 \pm 1.9$ months (range 13 hours-18 months), and it was significantly shorter in the stent grafting group than in the surgically treated group (9.6 \pm 9.1 days vs $4.8 \pm 4.1$ months, respectively; $P=.001)$.

There were 2 deaths (4.2\%) among the 48 patients of group II, both before aortic repair. The first patient was hemodynamically unstable (severely hypotensive) on arrival in the intensive care unit and lost more than $1500 \mathrm{~mL}$ blood through the chest drains. The CT scan showed contrast media extravasation (Figure 1). He died of rupture before arrival in the operating room for an emergency thoracotomy. The second patient was a 25-year-old woman with pelvic fracture and pulmonary contusion. She died 6 days after the accident of a massive pulmonary thromboembolism, as demonstrated by autopsy, despite anticoagulant therapy. The difference in mortality between groups I and II was statistically significant $(P=.04)$.

The major postoperative complications, such as paraplegia or acute renal failure, were only seen in group I and in patients in which no spinal cord protection was used (Table 4). One patient had coma secondary to diffuse cerebral ischemia. He had a laceration extending into the arch and was operated on with the aid of total CPB and hypothermic circulatory arrest. Three cases of left recurrent laryngeal nerve plasty occurred in group I. In group II, minor complications occurred only in patients who had undergone a surgical procedure: left recurrent nerve injury in 4 patients, pericarditis in 2 , and chylothorax in 1 .

\section{Endovascular Repair}

The endovascular stent procedure was successful in all cases. The devices used were the Talent stent graft (Medtronic, World Medical Corp, Sunrise, Fla) in 14 cases and the Excluder Thoracic endoprosthesis (W.L. Gore \& Associates, Inc, Flagstaff, Ariz) in 1. Digital subtraction angiography and transesophageal echocardiography revealed complete exclusion of the aneurysms in all cases without any primary leakage. In 5 patients, the left subclavian artery was partially covered by the proximal covered part of the graft. Digital subtraction angiography showed a reduced but persistent flow in the left subclavian artery in all but 1 patient. The patient with left subclavian occlusion did not show any functional prob- 
lem postoperatively or during follow-up. No other major complications occurred. All patients were discharged with oral $\beta$-blockers, and lifetime therapy was recommended. Clinical and imaging follow-ups with helical CT were scheduled at 1,6 , and 12 months after the procedure and yearly thereafter. Complete thrombosis of the aneurysmal sac and progressive fibrosis of the thrombosed aneurysms with reduction in size were observed in all cases at 6 months, and total aneurysmal shrinkage was complete at 12 months. We did not observe any alteration of the graft material with time, nor did we see a secondary endoluminal leakage during 7 years of follow-up.

\section{Discussion}

Delayed surgical repair in patients with TRTA resulted in a lower mortality than did immediate intervention. ${ }^{5-9}$ However, this strategy has not been readily accepted in clinical practice.

The impressive negative natural history of TRTA, according to the classic report of Parmley and coworkers, ${ }^{10}$ has induced us to consider this lesion as an absolute surgical emergency, but the combination of a cardiovascular intervention in a severely injured patient has resulted in an operative mortality ranging from $15 \%$ to between $45 \%$ and $50 \%$. Despite advances in surgical and resuscitation techniques in recent years, the perioperative and postoperative mortalities associated with TRTA have remained high. In our series, the mortality among patients undergoing emergency surgical repair was $19 \%$.

As pointed out by Pate and associates ${ }^{7,11}$ in several editorials and reports, the Armed Forces Institute of Pathology series reported by Parmley and coworkers ${ }^{10}$ does not apply to the current clinical reality. Moreover, a clear relationship between free aortic rupture and death was not reported, nor was it reported how much the other potentially fatal injuries, occurring in more than half of the patients, actually contributed to death. Nowadays, the risk of development of a delayed free aortic rupture is estimated to be considerably lower.

In most cases, a complete transection occurs, with instantaneous death. In about $15 \%$ of cases, the adventitial wall and mediastinal structures contain the rupture, allowing survival. ${ }^{10}$ In these cases, if adequate antihypertensive therapy acting to reduce wall stress is prompt, the risk of aortic rupture is limited. ${ }^{7}$

Hartford and colleagues ${ }^{12}$ reported on 86 autopsies performed on persons killed in vehicular crashes. Of these, 37 died at the scene of the accident, 16 arrived alive at the hospital, and only 1 died of complete aortic rupture. Similarly, Kalmar and coworkers ${ }^{13}$ reported an autopsy series of 168 subjects with TRTA in which 166 died within 2 hours of the accident and only 1 died of late rupture of a periaortic hematoma.
In a retrospective study, Pate and associates ${ }^{7}$ estimated that the risk of development of free rupture after arrival at the hospital was $4 \%$. Moreover, they showed that free rupture did not develop in any patient whose systolic blood pressure was maintained at less than $140 \mathrm{~mm} \mathrm{Hg}$ by drug therapy. ${ }^{7}$ Maggisano and colleagues ${ }^{8}$ demonstrated an operative mortality of $9 \%$ when using a more selective approach consisting of immediate repair for unstable patients and for stable patients with no contraindications to this early repair and deliberately delayed repair for patients with concomitant injuries or sepsis. In a group of 19 patients undergoing delayed repair, no deaths from aortic rupture were observed by Langanay and coworkers. ${ }^{14}$

Since 1992, we have delayed aortic repair in all patients who have arrived alive at the hospital unless signs of impending aortic rupture, such as hemodynamic instability, massive hemothorax, contrast media extravasation on CT, and rapid growth rate of pseudoaneurysm, were present. We had only 1 death from aortic rupture; this was in a patient with massive hemothorax and extravasation of contrast media on CT. A second death was related to massive pulmonary thromboembolisms despite anticoagulant therapy.

Recently, the development of endovascular techniques has provided additional opportunities in the treatment of descending aorta diseases, and the results of clinical studies have shown the feasibility of endovascular procedures in the treatment of traumatic aortic injury. ${ }^{15-20}$

Endovascular treatment requires some particular anatomic conditions; thus not all patients can be treated. At the least, proper peripheral vascular access is required. The most important anatomic characteristic of a posttraumatic lesion allowing endovascular treatment is the presence of an adequate proximal neck or at least $5 \mathrm{~mm}$ aortic wall from the subclavian artery with absence of mural thrombus, calcifications, or hemorrhage. Other studies have reported the artificial creation of an aortic neck, covering the left subclavian artery with the stent graft, with or without previous carotid-subclavian transposition. However, the curved anatomy of the aortic arch does not seem favorable for longterm efficacy of the stent graft, and the flow from the left subclavian artery may impede sealing of the aneurysm. The lifelong adjacency of the uncovered part of the stent graft to the left carotid artery is a potential source of emboli. Moreover, if symptoms from the closure of the left subclavian artery occur, and a carotid-subclavian bypass becomes necessary, the low invasiveness of the procedure is partially lost. In our endovascular series, 9 out of 15 patients had a short landing zone $(<1 \mathrm{~cm})$, but complete aneurysmal sealing was achieved in all cases.

Paraplegia may complicate the surgical repair in 3\% to $33 \%$ of cases, according to the literature. ${ }^{1,14,21,22}$ The risk of paraplegia decreases significantly when an active distal per- 
fusion system (such as total or partial CPB or left heart bypass) is used. ${ }^{1}$ Distal ischemic complications developed in 4 patients in group I: 3 cases of paraplegia ( 1 of these also with acute renal failure) and 1 case of paraparesis. No distal perfusion system had been used in any of them. CPB, either total or partial, requires full heparinization of the patients, which can induce fatal hemorrhage of brain, pulmonary, and abdominal contusions. This may possibly explain the higher mortality among patients operated on with total heparinization relative to those operated on without heparin in the meta-analysis of Van Oppell and colleagues. ${ }^{1}$ Total CPB should be limited to cases of lesions involving the aortic arch, where deep hypothermia and circulatory arrest are necessary to perform the proximal anastomosis.

To reduce the conflict between the necessary use of distal perfusion and the increased risk linked to full heparinization, the use of bypass systems with centrifugal pumps that do not make use of heparin are recommended. In group II, we performed all but 2 operations with the aid of a left heart bypass with a centrifugal pump without systemic heparinization, and major complications were not observed. At present, no case of paraparesis or paraplegia has been reported in the literature after the endovascular treatment of TRTA.

The endovascular technique does not require heparinization, carries a low invasiveness with attendant minimal blood loss, and can be applied in the acute phase without the risk of destabilizing pulmonary, head, or abdominal traumatic lesions. Moreover, standard sizes of thoracic stent grafts are available, allowing their use in an emergency. Stent-graft repair thus can be performed after trauma sooner than can surgical repair, soon after the management of other life-threatening lesions. In patients without severe associated lesions, delaying the treatment of TRTA does not provide any advantage, and it should be performed as soon as possible. The correct timing of aortic repair in a polytraumatized patient should be considered and balanced along with other severe injuries, without a fixed priority.

\section{Conclusion}

In conclusion, for many years, TRTA has been considered a highly lethal lesion and a potential cause of death in blunt chest trauma. Despite evidence in the literature of lower morbidity and mortality, initial medical management of uncomplicated aortic injury with subsequent delayed surgery has not been easily accepted in clinical practice. The development of endovascular techniques represents a viable alternative with very low risk and limited impact on trauma destabilization, even in the acute phase.

\section{References}

1. Von Oppell VD, Dunne TT, De Groot MK, Zilla P. Traumatic aortic rupture: twenty year metaanalysis of mortality and risk of paraplegia. Ann Thorac Surg. 1994;58:585-93.

2. Fabian TC, Richardson JD, Croce MA, Smith JS, Rodman G Jr, Kearney PA. Prospective study of blunt aortic injury: multicenter trial of the American Association for the surgery of trauma. J Trauma. 1996;42:374-383.

3. Kouchoukos NT, Dougenis D. Surgery of the thoracic aorta. $N$ Engl J Med. 1997;336:1876-88.

4. Tatou E, Steinmetz E, Jazayeri S, Benhamiche B, Brenot R, David M. Surgical outcome of traumatic rupture of the thoracic aorta. Ann Thorac Surg. 2000;69:70-3.

5. Galli R, Pacini D, Di Bartolomeo R, Turinetto B, Grillone G, Pierangeli A. Surgical indications and timing of traumatic ruptures of the thoracic aorta. Ann Thorac Surg. 1998;65:461-4.

6. Akins CW, Buckley MJ, Dagget W. Acute traumatic disruption of the thoracic aorta: a ten year experience. Ann Thorac Surg. 1981;31:305-9.

7. Pate JW, Fabian TC, Walker WA. Traumatic rupture of the aortic isthmus: an emergency? World J Surg. 1995;19:119-126.

8. Maggisano R, Nathens A, Alexandrova NA, Cina C, Boulanger B, McKenzie R, et al. Traumatic rupture of the thoracic aorta: should one always operate immediately? Ann Vasc Surg. 1995;9:44-52.

9. Kipfer B, Leupi F, Schuepbach P, Friedly D, Althause U. Traumatic rupture of the thoracic aorta: immediate or delayed surgical repair? Eur J Cardiothorac Surg. 1994;8:30-3.

10. Parmley LF, Mattingly TW, Marian WC. Non-penetrating traumatic injury of the aorta. Circulation. 1958;17:1086-100.

11. Pate JW. Is traumatic rupture of the aorta misunderstood? Ann Thorac Surg. 1994;57:530-1.

12. Hartford JM, Fayer RL, Shaver TE, Thompson WM, Hardy WR, Roys GD, et al. Transection of the thoracic aorta: assessment of a trauma system. Am J Surg. 1986;151:224-9.

13. Kalmar P, Otto CB, Rodewald G. Selection of the proper time for operation of traumatic thoracic aortic aneurysms (TTA). J Thorac Cardiovasc Surg. 1982;30:36-7.

14. Langanay T, Verhoye JP, Corbineau H, Agnino A, Derieux T, Menestret $\mathrm{P}$, et al. Surgical treatment of acute traumatic rupture of the thoracic aorta: a timing reappraisal? Eur J Cardiothorac Surg. 2002; 21:282-7.

15. Dake MD, Miller DC, Semba CP, Mitchell RS, Walker PJ, Liddell RP. Transluminal placement of endovascular of stent graft for the treatment of descending thoracic aortic aneurysms. N Engl J Med. 1994; 331:1729-34.

16. Grabewoeger M, Hutshala D, Ehrlich MP, Cartes-Zumelzu F, Thurnher S, Lammer J, et al. Thoracic aortic aneurysms: treatment with endovascular self- expandable stent grafts. Ann Thorac Surg. 2000:69: 441-5.

17. Fattori R, Napoli G, Lovato L, Russo V, Pacini D, Pierangeli A, et al. Indications for, timing of, and results of catheter-based treatment of traumatic injury to the aorta. AJR Am J Roentgenol. 2002;179:603-9.

18. Mitchell RS, Miller DC, Dake MD, Semba CP, Moore KA, Sakai T. Thoracic aortic aneurysm repair with an endovascular stent graft: the "first generation." Ann Thorac Surg. 1999;67:1971-4.

19. Fattori R, Napoli G, Lovato L, Grazia C, Piva T, Rocchi G, et al. Descending thoracic aortic diseases: stent-graft repair. Radiology. 2003;229:176-83.

20. Katz NM, Blackstone EH, Kirklin JW, Karp RB. Incremental risk factors for spinal cord injury following operation for acute traumatic aortic transection. J Thorac Cardiovasc Surg. 1981;81:669-74.

21. Nicolosi AC, Almassi HG, Bousamra M 2nd, Haasler GB, Olinger GN. Mortality and neurologic morbidity after repair of traumatic aortic disruption. Ann Thorac Surg. 1996;61:875-8.

22. Lachat M, Pfammatter T, Witzke H, Bernard E, Wolfensberger U, Kunzly A, et al. Acute traumatic aortic rupture: early stent-graft repair. Eur J Cardiothorac Surg. 2002;21:959-63. 